Vol. 122006 pp. 297-304

Andrzej Macioch

Chair of Cartography

E-mail: amacioch@uw.edu.pl

\title{
MODERN METHODS OF USE OF GEOGRAPHIC MAPS IN FIELD RESEARCH FOR LOCALISATION OF OBJECTS
}

\begin{abstract}
In the paper the author described briefly modern technical possibilities of supporting the use of maps in field research and presented methods of map use in localisation of objects. In his description of the methods the author presented the differences in the approach to the problem depending on the features of the maps, both paper and digital ones. He also stressed the issue of adjustment of the features related to the cartometricity of the maps in use to the coordinate system in which the data required for the field projects are obtained and processed.
\end{abstract}

Key words: field work, elements of mathematical basis, GPS, calibration, paper map, digital map, vector map, satellite receiver, geographic coordinates.

Geographic maps, as a specific model of the fragment of geographical space, are commonly accepted basic materials used in field research. It is worth noting that in field research both modern and old maps are used. Nowadays, and in particularly in the last decade, easily available satellite images and aerial photos became an excellent complement of maps. The latter turned out to be particularly useful after the popularisation (thanks to the development of computer science and technology) of orthophotomaps, which fully correspond to the classic large-scale maps as regards their application to measurements used to correctly determinate length, area or similar quantities.

The methodology of map use in field research has many aspects and creates many problems of both technical and academic nature. In this paper we will focus on one of its aspects, related to the basic function of the map: the function of orientation in space. This function includes the issue of determining the location of the investigator, of orientation with respect to geographic directions and the localisation with respect to other spatial elements situated both in proximity and at a distance.

A map can fulfil this function thanks to its isomorphism: of location, form and contents. For a map to fulfil this function appropriately, it should - apart from the necessary condition of correctness and sufficient accuracy of its 
content - contain visualised elements of its mathematical basis, first of all a graticule or at least a grid of planar orthogonal coordinates in a specific projection. A map without a graticule (or a grid of orthogonal plane coordinates) and used in the traditional way (without the use of a computer) does not ensure a high precision and accuracy in fulfilling its main function.

The use of such maps with help of computers, thus with help of appropriate software applications - browsers, graphic editors or GIS programs - is quite different; we will deal with this issue later.

As the title of the paper indicates, further discussion will deal with modern methods of map use in field research for the purpose of orientation in space. We should explain here what the methods consist in and how they differ from the ones used in the past.

The difference is namely in the possibility of applying, when using the map, new techniques of determining the position and of taking advantage of achievements of computer science and technology.

\section{MODERN TECHNICAL POSSIBILITIES}

Nowadays we have at our disposal excellent methods of position determination: the inertial method, whose high equipment costs and complex procedures of use became obstacles in its popularisation, and the satellite method, whose practical implementations are the systems NAVSTAR GPS (Global Positioning System), GLONASS (Global'naya Navigatsionnaya Sputnikovaya Sistema; Global Navigation Satellite System), as well as the European system GALILEO, still under construction. The systems listed here are described in detail in the literature in the fields of geodesy and navigation (Czarnecki K., 1994, Lamparski J., 2003); for that reason we will omit here their description. We only want to point the reader's attention to the fact that despite of their significant technical complexity they are very user-friendly. The simplicity of procedures of system use (at least as regards navigational applications - and such are of interest to us in field research) and a relatively low cost of receivers for position determination allowed for a significant popularisation of these systems, and in particular of the GPS, in recent years. The accuracy of position determination (except for special geodetic measurements) achieved by means of widely available navigational receivers range from around 20 metres to a few metres (with difference method). It would therefore seem that we finally have an efficient tool solving the ancient problem of position determination on the Earth. And this is indeed true, but the coordinates obtained from the measurement, although they determine unambiguously the position of the measurement point, do so in an abstract (for the investigator) coordinate system. They become concrete only in connection with the map regarded as a visualised model of a fragment of the geographic space. The coordinates themselves (geographic or planar orthogonal in the specified cartographic projection), although 
they constitute valuable data allowing for calculations describing certain relations among elements in space, do not - when detached from the map - constitute readable information about orientation in space in field research. Because of this, most modern satellite receivers are equipped with liquid crystal display screens, often colour ones, which make it possible to display the map stored in the memory of the receiver. In the case of portable, very small receivers, however, the quality of the map image is not satisfactory. The situation is different in the case of car or plane receivers, but their usability in field research is low, due to their much larger dimensions as compared to portable receivers.

In recent years portable computers such as laptops, notebooks and palmtops (pocket PCs) became another important element of field research with a map, apart from the use of satellite systems of position determination. Such computer systems, nowadays not much less powerful than desktops (not counting, of course, workstations and special computers), can be used for several hours with a battery. Moreover - as it is the case with some notebooks - they can be equipped with screens which allow for use in bright daylight, which makes them an excellent tool for use in field research. Computers, however, are useless if they don't have application programs appropriate for the specific tasks. In our case, the relevant software consists of graphic editors allowing for representation of the map both in vector and in raster form. The necessary condition for the usability of the application in question is map editing in the specific coordinate system. Therefore editors such as Corel Draw or Corel Photo Paint, otherwise excellent, are here entirely useless. A very desirable feature of such editors is the possibility of inputting data from satellite receivers, which is usually possible when the receiver and the computer are connected with a cable through a serial port (RS 232 or USB) or without a cable by means of Bluetooth. The import of data from the receiver allows for imaging in real time, on the background of the map, of a point where the measurement takes place. Such connection of the map with data from satellite measurements allows the fieldwork investigator to visualise unambiguously his spatial location both in absolute and relative aspect.

Modern laser rangefinders not requiring mirrors, electronic compasses, inclinometers and altimeters are valuable complementary devices complementing the above-mentioned elements and demonstrating a modern approach to field research. Such measuring instruments are often integrated into one device, which makes the fieldwork extremely efficient. There are many technical solutions. Since their analysis is not the goal of this paper, we will omit their description. We will only mention that these devices can be utilised, among other things, to determine the position of inaccessible or difficult to access objects - a problem that until recently was solved by means of work-intensive methods known from the traditional topography or geodesy.

Summarising, we can state that while until recently in field research we had at our disposal only paper map, compass, barometric altimeter and a simple inclinometer, nowadays we have (at least potentially): 
- paper map,

- digital map in form of raster image,

- vector map,

- computer (tablet, laptop or similar portable device) and appropriate application software,

- receiver for satellite measurements (mostly in the GPS system),

- laser rangefinder equipped in electronic compass and inclinometer,

- altimeter (built into a satellite receiver or as a stand-alone device).

A minor remark: When writing about the use of satellite receivers we have in mind receivers working in the GPS system, since at the time of writing this paper this is the fundamental system in practice.

\section{METHODS}

The variety of available technical methods presented above, although they eventually expand our abilities to use maps in field research, complicate somewhat the process of their use. In further discussion we will present selected issues related to the methods of map use in field research for spatial orientation, as was already mentioned.

Analysing available geographic maps (both paper and digital - of vector or raster type) we can divide them into four subsets:

- maps containing elements of mathematical basis in a known system, described by coordinate values,

- maps containing elements of mathematical basis in an unknown system, described by coordinate values,

- maps not containing elements of mathematical basis in an unknown system, described by coordinate values but whose mathematical basis is known,

- maps not containing elements of mathematical basis in an unknown system, described by coordinate values and whose mathematical basis is unknown.

From the point of view of the method of map use, the second and fourth subsets can be treated as one - maps not containing data about the location of the elements of their content in a specific coordinate system. The third subset is close to them and in many case it can be treated in the same way, but in some other cases we are able, if we have other cartographic materials at our disposal, to complement the data related to their mathematical basis and give them certain properties of the first subset. In general, we can simplify things a little and in further discussion we can use a two-subset division into maps with elements of mathematical basis described by coordinates in a known systems and maps lacking this information.

The use of paper maps contained in the first group causes no problems. When the values of coordinates read from the satellite receiver are con- 
verted into the values in the map system, they allow for unambiguous localisation of the measurement point on the map.

If the object to be localised on the map is physically inaccessible but visible, we have to perform a more complicated measurement. Namely, we have to measure the distance from the point in which the satellite measurement is possible or whose coordinates we known (e. g., it is identifiable on the map) to the measured object as well as the azimuth towards this object, which will allow us to calculate the coordinates of this object. Knowing, in turn, the altitude of the point from which the measurement is being performed, we can obtain the difference in altitude by measuring, with an inclinometer, the vertical angle of the direction toward the measured object. Therefore we can obtain also the altitude of the point of object localisation. In this case an excellent device is a reflectorless laser rangefinder with an integrated electronic compass and inclinometer.

The author of this paper conducted a measurement experiment using the laser rangefinder LaserAce 300 made by MDL with built-in inclinometer and electronic compass allowing for the measurement of the magnetic azimuth. The range of distance measurement of this rangefinder without the use of a prismatic reflector reaches $300 \mathrm{~m}$. The error of the location of the point measured was equal to $\pm 5 \mathrm{~m}$ at the distance of $300 \mathrm{~m}$ and its distribution was a band up to $20 \mathrm{~cm}$ wide, perpendicular to the direction of the measurement. Of course it is possible to achieve a greater accuracy using a different measuring instruments.

Use of paper maps lacking the elements of mathematical basis described by coordinates in a known system makes it impossible to localise objects based on data from measurements performed by a satellite receiver and is nowadays an anachronism. Of course, using procedures of map orientation and determination of the current position known from topography, we can localise on the map the point where we are now; this method, however, is far from modern.

It is definitely more convenient to replace the use of a paper map in the classic manner, even when utilising data from a satellite receiver, by computer support. For this purpose we have to use a vector or raster map instead of a paper map. Usually, access to vector maps is difficult, therefore most often we will have at our disposal a digital map in form of a raster. Nowadays everybody with a paper map and access to a scanner (and, of course, has the permission of the copyright owner of the map for such use) can prepare such map.

The inclusion of computer technology into the process of utilisation of a map definitely changes this process. First of all, the above-mentioned classification of maps according to the criterion of mathematical basis is no more as important as it used to be. Why this is so will be seen from later discussion. Furthermore, when appropriate application software is used, import of data from a satellite receiver becomes possible, and therefore also visualisation and registration of the location of the point being measured, 
of the direction in which the person performing field research moves, as well as the registration of the route of his movement during the research. All these elements can be represented graphically on the basis of the map read into computer memory.

When writing about the application of computer technology, we assume that the person conducting field research has appropriate software at his disposal. From the methodological point of view, it is worthwhile to mention the features that the software should have. It should namely ensure:

- editing of the map in the specific coordinate system; the set of available systems should contain these systems in which we intend to perform our work;

- in the case of vector map use, the possibility of transformation of the vector map into a specific system, and in the case of raster map use, the possibility of the so-called calibration of the raster image of the map, that is, the transformation of the raster image to the form consistent (in cartometric respect) with the image in the specific coordinate system;

- the possibility of importing data from satellite receivers by means of two protocols: the standard one, accessible for all GPS receivers marked as NMEA and (although this is not necessary) GARMIN for the devices made by GARMIN, a leading company in the field of production of navigational receivers;

- possibility of visualisation and registration of positions received from the receiver.

The necessary condition for the correctness of cooperation of the individual components of the system: map - computer - satellite receiver - other device capable of exporting spatial data is their operation in the same coordinate system. Of course, data obtained from the satellite receiver and other measurement instruments can be transformed to a specific system as they are being obtained, but the map must be edited after the reduction to the desired system.

For clarity in our discussion, let's call the coordinate system in which the map is worked out the "map system", the system used in field research, the "work system" and the system in which data are exported from the receiver, the "receiver system".

The analysis of technical characteristics of satellite receivers operating in the GPS system allows for introduction of certain simplification in our discussion. Namely, we can assume that the work system is consistent with the receiver system. This follows from the installation, in computers embedded in satellite receivers, of transformation definitions and procedures of the majority of the coordinate systems used in practice - both of geographic (geodesic) system and of orthogonal planar coordinates (in a specified mapping). Therefore, the user of the receiver can export from it coordinates in the chosen system.

In the case of vector maps we can distinguish two cases: 
In the first case, the map system is consistent with the work system. This situation makes the use of the map very convenient, since it requires only that the map be input into the appropriate program satisfying the above conditions.

In the other case, the map is used in the system other than the work system. This situation requires that the transformation procedure of the vector image of the map to the work system be transformed. As we already know, transformation procedures require data defining relations among the systems occurring in the transformation process. In our case, if we don't have such relations, we can determine them by measuring the coordinates of the control points, which will be done using, of course, GPS satellite receivers.

When deciding to use maps which require performing transformation procedures we should be aware that the transformation performed may reduce the accuracy of the maps.

When using raster images of maps we can't omit the transformation procedures; with regard to raster images, such procedures are usually called calibrations. The calibration of such image is a very important procedure from the point of view of map use, since it allows not only for bringing the map to the form consistent with the image in the specified coordinate system, but also for eliminating, partly or even entirely, deformations of the scanned original and distortions formed during the scanning process (resulting from the faulty operation of the scanner).

Depending on the degree of raster image transformation, for the realisation of this procedure it is necessary to have the coordinates of the appropriate number of control points. For instance, for the Helmert transformation two points suffice; for the affine model, three; for bilinear, four; for biquadratic, nine; and for bicubic, 16. The degree of transformation depends of course on the degree of image deformation and on the change of the cartographic mapping with respect to the mapping, in which the scanned map was made.

The coordinates of the control points may be:

- read directly from the image of the map used, if the map contains elements of mathematical basis described by coordinate values (e. g., graticule or the grid of planar orthogonal coordinates);

- obtained from other cartographic or geodesic materials;

- obtained by measurement from a satellite receiver with a possible extension of the measurement by use of the above-mentioned procedure of determination of inaccessible points (e. g., using a rangefinder and compass).

A good - in author's opinion - example of a program allowing for the calibration of the raster and its further utilisation to work with the map on computers of various types, from desktops to pocket computers (palmtops) is OziExplorer. Of course many other application software packages made for creation of geographic information systems include both a calibration 
function for raster image and capability for importing data from GPS receivers, but these programs are more inconvenient for use in field work.

As follows from the discussion, the application of computer technology, advanced procedures of raster image transformation, possibility of performing not very complicated satellite procedures for determination of position on the Earth, makes possible the full use of map in field research; the problem of localisation of the objects under investigation was until recently extremely difficult or even impossible - at least with sufficient accuracy.

\section{REFERENCES}

Czarneck i K., 1994, Geodezja wspótczesna w zarysie [Outline of Modern Geodesy; in Polish], Wydawnictwo Wiedza i Zycie, Warszawa.

Lam parski J., 2003, GPS $w$ geodezji [GPS in Geodesy; in Polish], Gall s.c. O. Graca, M. Ziętek, Katowice

English translation: Matgorzata Mikulska 